\title{
Preferência do paciente no rastreamento do câncer colorretal: uma comparação entre colonografia por tomografia computadorizada e colonoscopia*
}

Patient preferences toward colon cancer screening: a comparison between computed tomography colonography and conventional colonoscopy

\author{
Marcos Vinicius Alvim Soares Maia ${ }^{1}$, Augusto Castelli von Atzingen², Dario Ariel Tiferes ${ }^{3}$, \\ Sarhan Sydney Saad ${ }^{4}$, Elisabeth Deak ${ }^{5}$, Delcio Matos ${ }^{6}$, Giuseppe D'lppolito ${ }^{7}$
}

Resumo Objetivo: Avaliar o grau de aceitação do paciente submetido a colonografia por tomografia computadorizada (CTC) em comparação com a colonoscopia, quando realizadas para rastreamento de doença colorretal. Materiais e Métodos: Cinquenta pacientes com suspeita de doença colorretal foram submetidos a CTC e colonoscopia. Questionários foram aplicados antes e após a realização da CTC e após a colonoscopia. Graduou-se o desconforto esperado e experimentado antes e após a realização da CTC e da colonoscopia, bem como a preferência do paciente por exame. Resultados: Em relação à CTC, antes de iniciar o exame 18\% dos pacientes afirmaram esperar pouco desconforto, $78 \%$, desconforto moderado e $4 \%$, muito desconforto. Após a realização do exame, $72 \%$ dos pacientes relataram pouco desconforto, $26 \%$, desconforto moderado e apenas um (2\%) dos pacientes referiu muito desconforto. Após a realização da colonoscopia, $86 \%$ dos pacientes relataram preferência pela CTC. 0 grau de distensão colônica e a quantidade de fluido residual não influenciaram na preferência dos pacientes. Conclusão: Os pacientes preferiram a CTC à colonoscopia, não havendo relação estatística com o grau de distensão colônica na CTC e a eficiência do preparo intestinal. Unitermos: Colonoscopia; Colonoscopia virtual; Colonografia por tomografia computadorizada; Tomografia computadorizada; Preferência do paciente; Satisfação do paciente.

Abstract Objective: To assess the degree of acceptance of patients undergoing computed tomography colonography (CTC) in comparison with colonoscopy in the screening of colorectal disease. Materials and Methods: Fifty patients with suspected colorectal disease underwent CTC and colonoscopy. Questionnaires were administered before and after the performance of the CTC and after the colonoscopy. The discomfort expected and experienced before and after the performance of both procedures as well as the patients' preference for each method were evaluated. Results: As regards CTC, before the procedure, $18 \%$ of the patients reported expecting little discomfort, $78 \%$, mild discomfort, and $4 \%$, a lot of discomfort. After the procedure, $72 \%$ of the patients reported little discomfort, $26 \%$, mild discomfort, and only one (2\%) of the patients reported a lot of discomfort. Upon completion of the colonoscopy, $86 \%$ of the patients reported their preference for CTC. The degree of colonic distention and residual amount of fluid had no influence on the patients' preference. Conclusion: CTC was preferred to colonoscopy, with no statistical relationship with the degree of colonic distention at CTC and efficiency of bowel preparation.

Keywords: Colonoscopy; Virtual colonoscopy; Computed tomography colonography; Computed tomography; Patients' preference; Patients' satisfaction.

Maia MVAS, von Atzingen AC, Tiferes DA, Saad SS, Deak E, Matos D, D'Ippolito G. Preferência do paciente no rastreamento do câncer colorretal: uma comparação entre colonografia por tomografia computadorizada e colonoscopia. Radiol Bras. 2012 Jan/Fev; 45(1):24-28.

* Trabalho realizado no Departamento de Diagnóstico por Imagem da Universidade Federal de São Paulo (Unifesp), São Paulo, SP, Brasil.

1. Médico Radiologista, Especializando do Setor de Abdome do Departamento de Diagnóstico por Imagem da Universidade Federal de São Paulo (Unifesp), São Paulo, SP, Brasil.

2. Médico Radiologista, Pós-graduando nível Doutorado em Radiologia Clínica da Universidade Federal de São Paulo (Unifesp), São Paulo, SP, Brasil.

3. Doutor, Médico Radiologista Colaborador do Departamento de Diagnóstico por Imagem da Universidade Federal de São Paulo (Unifesp), São Paulo, SP, Brasil.

4. Professor Adjunto da Disciplina de Gastroenterologia Cirúrgica da Universidade Federal de São Paulo (Unifesp), São Paulo, SP, Brasil.
5. Médica Assistente da Disciplina de Gastroenterologia Cirúrgica da Universidade Federal de São Paulo (Unifesp), São Paulo, SP, Brasil.

6. Professor Titular da Disciplina de Gastroenterologia Cirúrgica da Universidade Federal de São Paulo (Unifesp), São Paulo, SP, Brasil.

7. Professor Livre-Docente do Departamento de Diagnóstico por Imagem da Universidade Federal de São Paulo (Unifesp), São Paulo, SP, Brasil.

Endereço para correspondência: Dr. Giuseppe D'Ippolito. Rua Professor Filadelfo Azevedo, 617, ap. 61, Vila Nova Conceição. São Paulo, SP, Brasil, 04508-011. E-mail: giuseppe_dr@uol. com.br

Recebido para publicação em 20/12/2011. Aceito, após revisão, em 17/1/2012.

\section{INTRODUÇÃO}

A detecção e consequente ressecção de carcinomas colorretais na fase precoce ou de lesões precursoras adenomatosas podem reduzir consideravelmente a morbidade $\mathrm{e}$ mortalidade por câncer colorretal, todavia, as taxas de participação da população em geral no rastreamento do carcinoma colorretal estão abaixo do desejável ${ }^{(1-3)}$. Embora o rastreamento tenha aumentado entre 
1987 e $1992^{(4)}$, a adesão às recomendações adotadas pelas principais sociedades clínicas está longe de ser satisfatória ${ }^{(5)}$. O sucesso de um programa de rastreamento depende basicamente de fatores socioeconômicos, da sua aceitação pela população e pela comunidade médica, e da adoção de políticas públicas eficientes ${ }^{(\mathbf{6 - 9})}$. Novas estratégias baseadas em tecnologias avançadas podem aproximar ou afastar a populaçãoalvo do programa de rastreamento, em função da sua percepção quanto aos custos e desconforto em usar determinado teste ${ }^{(6)}$.

Os métodos mais utilizados na detecção de pólipos e do carcinoma colorretal são a pesquisa de sangue oculto nas fezes, a sigmoidoscopia, o enema baritado e a colonoscopia $^{(2)}$. Para um exame completo do cólon, tanto o enema baritado como a colonoscopia podem ser realizados. A colonoscopia é mais eficaz que o enema baritado na detecção de pólipos e, além disso, oferece a possibilidade da realização de biópsia e remoção das lesões ${ }^{(4)}$. Entretanto, a colonoscopia é invasiva e tem algumas importantes desvantagens, como a dor e o desconforto referidos pelos pacientes, frequentemente sendo necessárias sedação e analgesia. Além disso, a colonoscopia está associada com risco de perfuração, embora pequeno, de até $0,9 \%{ }^{(4)}$, podendo também ocorrer dificuldade na progressão do aparelho, com consequente falha na visualização de todo o cólon em $5 \%$ a $15 \%$ dos pacientes (denominada de colonoscopia incompleta $)^{(\mathbf{5})}$.

Nas últimas décadas, a colonografia por tomografia computadorizada (CTC) ou colonoscopia virtual tem despontado como alternativa à colonoscopia óptica no rastreamento do carcinoma colorretal, sendo adotada, recentemente, por diversas sociedades médicas ${ }^{(8)}$. A CTC é um exame tomográfico baseado em múltiplos cortes axiais finos e reformatados em duas e três dimensões, incluindo perspectivas endoluminais do cólon, semelhantes às obtidas na colo$\operatorname{noscopia}^{(\boldsymbol{9})}$. É um procedimento rápido e eficiente (dura geralmente menos de dez minutos), reprodutível, com elevada acurácia, e bem tolerado pelo paciente, pois dispensa o uso de medicamentos durante a sua realização, além de ser minimamente invasivo, utilizando-se apenas a sondagem retal e insuflação com ar ambiente ${ }^{(\mathbf{1 0 - 1 2})}$. O paciente não é submetido a nenhum tipo de sedação e pode retomar às suas atividades imediatamente após o término do exame ${ }^{(7)}$. Na última década, a CTC tem sido introduzida no nosso meio e progressivamente adotada para o rastreamento do carcinoma colorretal e nos pacientes com colonoscopia incompleta, com resultados promissores, mas ainda pouco divulgados. Considerando que a sensibilidade da CTC para pólipos maiores que $6 \mathrm{~mm}$ é semelhante à da colonoscopia ${ }^{(10)}$ e que a taxa de complicações inerentes ao método é menor ${ }^{(\mathbf{9})}$, um importante fator de adesão à CTC seria a sua aceitação pelo paciente se for considerada mais bem tolerada que outros métodos diagnósticos, como a colonoscopia. Assim, a CTC poderia ser plenamente incorporada entre as ferramentas diagnósticas para o rastreamento do carcinoma colorretal. A tolerância à dor e ao desconforto está intrinsecamente ligada a fatores socioculturais e econômicos ${ }^{(\mathbf{1 3})}$, não sendo pertinente adotar completamente conclusões baseadas em estudos realizados em outros contextos. $\mathrm{Na}$ literatura nacional não encontramos dados que estabeleçam o grau de tolerância de pacientes submetidos à CTC no nosso meio.

A proposta do presente estudo foi avaliar o grau de aceitação e preferência entre a CTC e a colonoscopia, em pacientes submetidos a rastreamento de doença colorretal, e em função do seu preparo intestinal e grau de distensão intestinal.

\section{MATERIAIS E MÉTODOS}

Este estudo é uma pesquisa clínica tipo secundária, prospectiva, transversal realizada em um único centro e aprovada pela Comissão de Ética em Pesquisa da nossa Instituição. Foram realizados exames de CTC e colonoscopia em 50 pacientes (27 mulheres e 23 homens) com idade entre 36 e 82 anos (média de 61,06 anos), pré-agendados para realização de exames de colonoscopia, conforme rotina do Serviço de Endoscopia Digestiva, no período de setembro de 2010 a outubro de 2011. Todos os pacientes foram submetidos a um questionário especificamente construído para este fim, antes e após a realização da CTC e da colonoscopia.

Foram considerados critérios de inclusão na pesquisa: pacientes maiores de 18 anos, com suspeita de doença colorretal. Os critérios de exclusão foram: pacientes menores de 18 anos, gestantes, pacientes com quadro de doença diverticular aguda nas últimas duas semanas, ressecção de pólipos nas últimas seis semanas, cirurgia colônica prévia, pacientes sem condições clínicas de realizar o preparo colônico prévio e os sem condições cognitivas para responder ao questionário.

O questionário avaliou o preparo intestinal, os efeitos adversos ao preparo e o grau de desconforto esperado e experimentado pelo paciente antes e após o exame, numa escala de três pontos (pouco desconforto, desconforto moderado e muito desconforto). Após a colonoscopia, foi pedido ao paciente comparar o desconforto decorrente da colonoscopia em relação à CTC, estabelecendo a sua preferência para um determinado exame.

O preparo para o exame de CTC foi idêntico ao utilizado para a colonoscopia. Todos os pacientes ingeriram, na noite precedente ao exame, dois comprimidos de bisacodil, além de $1.500 \mathrm{ml}$ de água com dez envelopes de macrogol, com a adição de um frasco de $50 \mathrm{ml}$ de contraste iodado hidrossolúvel. Foi considerado adequado o preparo intestinal que proporcionasse uma limpeza colônica completa, com a menor quantidade de resíduo fecal possível, assim como a menor quantidade de fluido residual, possibilitando, portanto, uma maior confiança para o radiologista e a navegabilidade pelo programa de colonoscopia virtual (Figura 1).

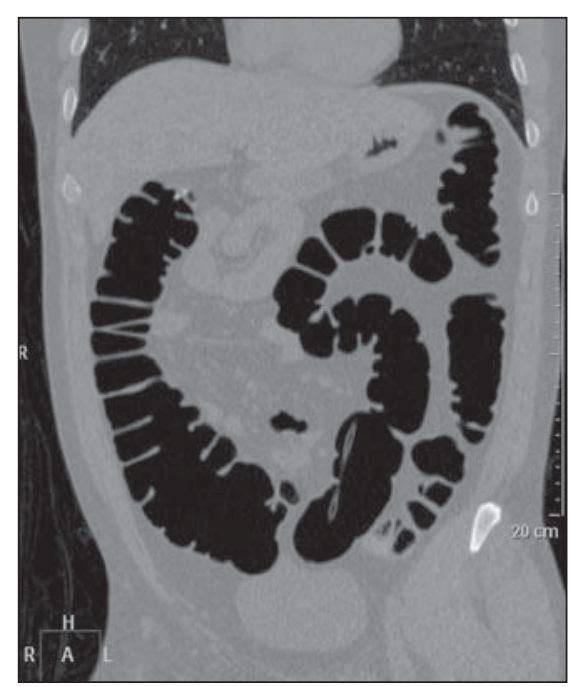

Figura 1. Reconstrução coronal mostrando excelente distensão de toda a moldura cólica. 
Todos os exames foram realizados em equipamento de tomografia computadorizada helicoidal com multidetectores de 64 fileiras (Brilliance; Philips Medical Systems, Best, Holanda) e equipado com programa de navegação colônica virtual.

Os exames foram realizados com colimação de $64 \times 0,625 \mathrm{~mm}$, tempo de rotação de $0,5 \mathrm{~s}$, espessura de corte de $2 \mathrm{~mm}$, espessura de reconstrução de $1 \mathrm{~mm}$, pitch de 1.1, baixa dose de radiação (120 kV e $50 \mathrm{mAs})$, estendendo-se o estudo do diafragma até a sínfise púbica. Não foram utilizados antiespasmódicos ou outras medicações.

Durante a realização da CTC, foi insuflado ar ambiente pelo reto por intermédio de sonda retal, de acordo com a tolerância do paciente e a qualidade de imagem, com o objetivo de se obter insuflação e distensão intestinal adequadas. Todos os exames foram realizados adquirindo-se imagens em decúbitos ventral e dorsal.

Todos os pacientes foram submetidos à colonoscopia imediatamente após a CTC, utilizando-se um videocolonoscópio adulto (Olympus CF-VL; Olympus Optical do Brasil, São Paulo, Brasil) e sob sedação (meperidina e midazolam). Após a colonoscopia, um novo questionário foi proposto aos pacientes com o objetivo de se estabelecer a sua preferência, quando comparada com a CTC previamente realizada.

Mediante a análise de variáveis responsáveis pela qualidade do exame de CTC, como o grau de distensibilidade do cólon (grau 1: não distendido; grau 2: distensão adequada; grau 3: distensão excelente) e a quantidade de fluido intestinal residual (ocupando mais ou menos que $50 \%$ da luz do cólon), procuramos correlacionar o grau de desconforto referido pelo paciente e sua preferência (Figuras 2, 3 e 4).

\section{Análise estatística}

Os dados categóricos foram resumidos em termos de frequência absoluta (número de pacientes) e relativa (porcentagem). Para a comparação entre duas variáveis que admitem o mesmo tipo de resposta, foi utilizado o teste de McNemar para verificar a significância das mudanças. Para verificar a associação entre duas variáveis categóricas quaisquer, foi aplicado o teste exato de Fisher, de modo que os dados pudessem ser transformados numa tabela do tipo $2 \times 2$ para a aplicação do teste.

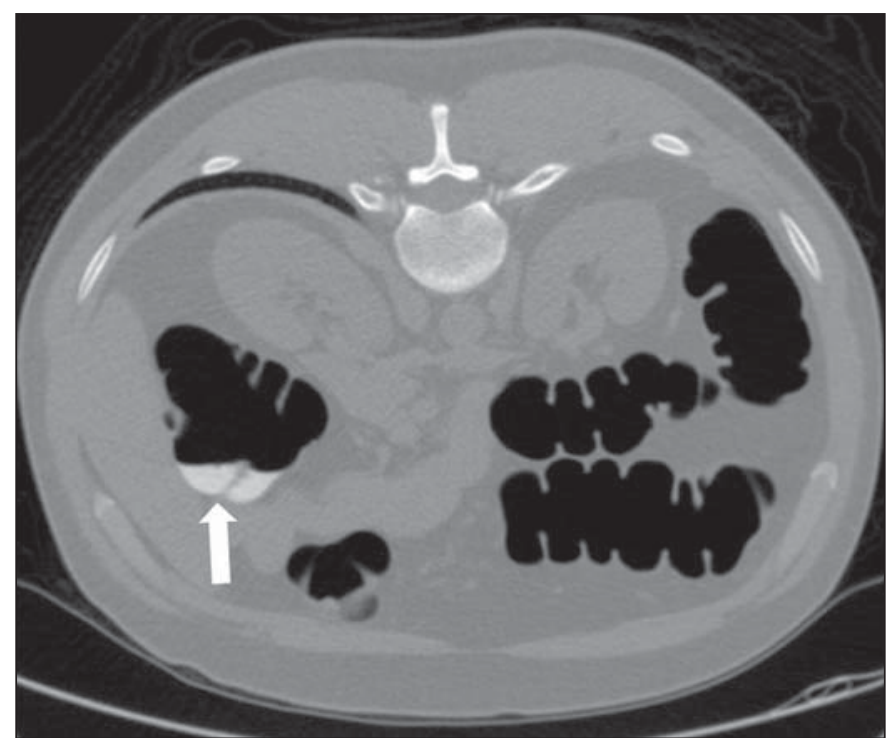

Figura 2. Paciente em decúbito ventral, com a quantidade de fluido residual menor que $50 \%$ da luz do cólon (seta).

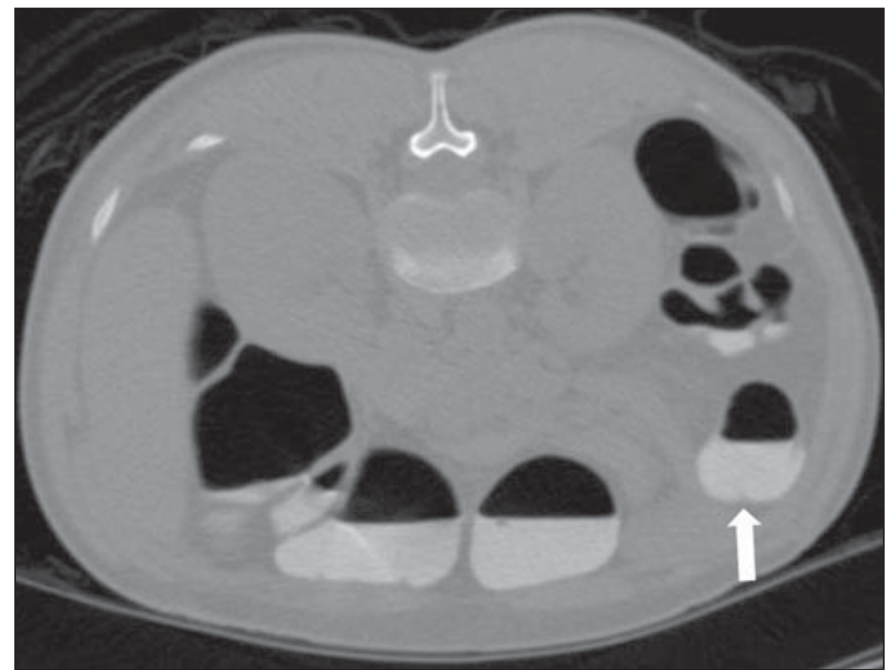

Figura 3. Imagem de paciente em decúbito ventral mostrando a quantidade de fluido residual ocupando mais que $50 \%$ da luz do cólon (seta).

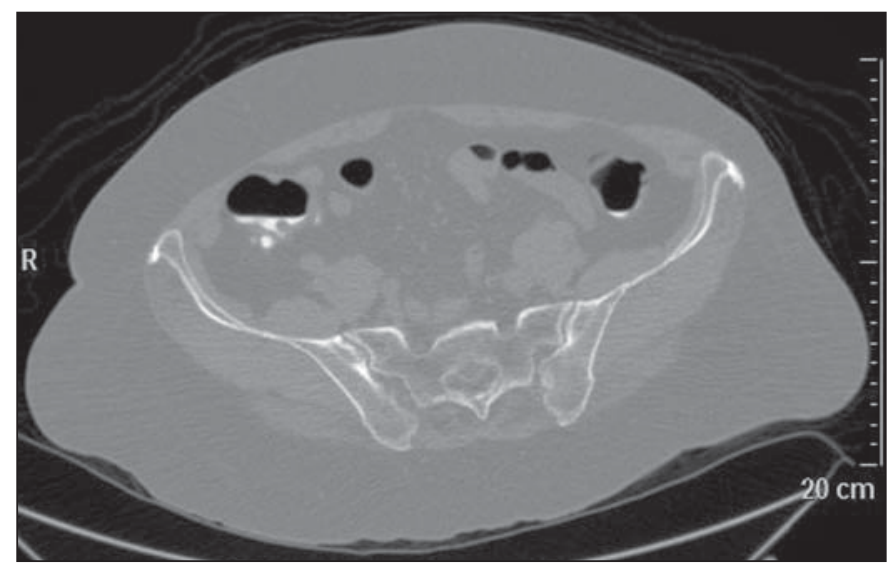

Figura 4. Exemplo de estudo com distensão inadequada, por distensão incompleta do cólon. 
Significância estatística foi estabelecida para valores de $p<0,05$. Foi utilizado o software estatístico Minitab ${ }^{\circledR}$, versão 16.1, para a análise dos dados.

\section{RESULTADOS}

O preparo intestinal foi considerado adequado em 44 pacientes $(88 \%)$. Seis pacientes $(12 \%)$ apresentaram preparo intestinal inadequado porque ingeriram parcialmente ou não ingeriram o contraste oral. Os efeitos colaterais ao preparo intestinal foram raros e relatados apenas por um paciente $(2,0 \%)$ que apresentou vômitos durante o período do preparo intestinal.

\section{Desconforto na CTC}

Após a realização do exame, 36 (72\%) pacientes relataram pouco desconforto, 13 (26\%) assinalaram desconforto moderado e um $(2 \%)$ paciente referiu muito desconforto. Trinta e um $(62 \%)$ pacientes relataram que o desconforto foi menor do que esperavam, sendo que 29 esperavam desconforto moderado e durante o exame afirmaram que tiveram pouco desconforto.

Dezesseis (32\%) pacientes disseram que o desconforto foi o esperado, sendo que seis julgavam inicialmente que teriam pouco desconforto e 10 que teriam desconforto moderado. Somente três $(6 \%)$ pacientes referiram um grau de desconforto na CTC maior do que o esperado.

A sensação de desconforto experimentada pelos pacientes em relação ao exame de CTC foi significativamente menor do que o esperado, de acordo com o teste de McNemar $(p<0,001)$.

\section{Avaliação da relação entre a preferência dos pacientes em relação ao tipo de exame e o grau de distensão do cólon e presença de fluido residual}

Quarenta e três pacientes (86\%) preferiram a CTC em relação à colonoscopia e somente seis pacientes (12\%) preferiram a colonoscopia óptica. Um dos pacientes (2\%) não expressou preferência.

A preferência dos pacientes por este ou aquele exame não parece estar relacionada com o grau de distensão do cólon ( $p=$ $0,999)$ e a quantidade de resíduo líquido intestinal ( $p=0,310)$, de acordo com o teste exato de Fisher.
Avaliação da relação entre o desconforto experimentado pela CTC com a distensão do cólon e o fluido residual

Não houve relação estatisticamente significante entre o desconforto relacionado ao exame de CTC e o grau de distensão do cólon ( $p=0,320)$. Também não houve relação estatística significante entre o desconforto e a quantidade de fluido residual $(p=0,414)$.

\section{DISCUSSÃO}

Programas de rastreamento são a principal maneira de reduzir a morbimortalidade por câncer colorretal. A colonoscopia tem sido, junto com a pesquisa de sangue oculto nas fezes e de antígeno carcinoembrionário, as principais ferramentas de rastreamento $^{(2)}$.

A principal vantagem da colonoscopia é a possibilidade de realização de biópsia e polipectomia quando necessário. Apresenta limitações como o custo mais elevado que outros métodos ${ }^{(8)}$, alguma morbidade e a apreensão e insegurança do paciente em relação à sedação e ao desconforto do exame ${ }^{(\mathbf{1 4 , 1 5})}$. Além disso, apesar de ser considerado o padrão de referência na detecção de lesões colônicas, alguns estudos relatam falha na detecção de grandes adenomas em até $12 \%$ dos $\operatorname{casos}^{(\mathbf{1 4})}$.

A CTC tem sido proposta como uma alternativa viável, com eficácia semelhante à da colonoscopia e vantagens relacionadas à rapidez do exame, menor invasividade, dispensando a necessidade de sedação e permitindo ao paciente retornar às suas atividades logo após a realização do procedimento $^{(\mathbf{8 , 1 0 )}}$.

Diversos trabalhos têm comparado a CTC à colonoscopia no que se refere à aceitação do paciente. Um estudo recentemente publicado demonstrou que $72 \%$ dos pacientes submetidos a CTC e colonoscopia preferiram a primeira ${ }^{(16)}$. Um outro estudo realizado em Missouri, EUA, aplicando em indivíduos submetidos a CTC e colonoscopia no mesmo dia um questionário semelhante ao utilizado no nosso trabalho, revelou que $68 \%$ dos entrevistados consideraram a CTC mais conveniente e que a preferiria para futuros controles ${ }^{(17)}$.

Na nossa revisão bibliográfica encontramos apenas um estudo que demonstrou que havia pacientes mais satisfeitos com a colonoscopia $^{(\mathbf{1 8})}$. A justificativa para isto parece estar relacionada com o ambiente em que a colonoscopia foi realizada, sendo que durante o procedimento o paciente estava em contato mais próximo com o médico e o enfermeiro ${ }^{(\mathbf{1 8})}$. No nosso estudo, a interação com o médico radiologista antes, durante e após a realização da CTC, para esclarecimento de eventuais dúvidas, deixou o paciente mais seguro e confiante, aumentando assim, possivelmente, a preferência pela CTC.

Uma questão a ser considerada neste tipo de pesquisa é o conhecimento do paciente em relação à limitação da CTC em biopsiar e ressecar pólipos intestinais, quando comparada com a colonoscopia. Neste sentido, esta informação poderia mudar a preferência dos pacientes, relativizando o desconforto experimentado, desde que a probabilidade de ocorrência de lesão colônica clinicamente significante fosse expressiva. Estudos de rastreamento mostram uma taxa de detecção de pólipos grandes (> $10 \mathrm{~mm})$ de $5 \%$ a $6 \%$ e de pólipos pequenos $(6-9 \mathrm{~mm})$ de $8 \%{ }^{(\mathbf{1 5})}$. A prevalência de adenomas avançados com risco de transformação maligna é de $0,5 \%{ }^{(15)}$, ou seja, provavelmente em apenas uma a cada 20 colonoscopias será necessária a ressecção de pólipos intestinais. Um consenso recente propôs que pacientes com dois pólipos menores que $10 \mathrm{~mm}$ devem fazer controle com CTC após três anos, em vez de se submeter imediatamente à polipectomia, que é recomendada para pólipos grandes (> $10 \mathrm{~mm}$ ) ou se três pequenos pólipos estiverem presentes ${ }^{(\mathbf{1 9})}$.

No nosso trabalho foi possível estabelecer a preferência dos pacientes pela CTC, apesar do número relativamente pequeno de indivíduos pesquisados e que deverá ser expandido em próximos estudos. Além da pequena casuística, o nosso trabalho apresenta outras limitações: a) a avaliação é subjetiva e pode ser influenciada pelo pesquisador, o que procurou ser evitado, permitindo ao paciente responder de próprio punho ao questionário; b) a proximidade do radiologista encarregado da pesquisa com o paciente durante as diversas fases do estudo pode ter contribuído para gerar empatia e tendência a se considerar o exame de CTC mais confortável. 
Por outro lado, os nossos resultados demonstraram que a preferência dos pacientes não está relacionada com a distensão colônica na CTC e a eficiência do preparo intestinal. Para isto, deve ter contribuído o fato de a insuflação ter sido realizada tomando-se o cuidado de não exceder a tolerância do paciente, porém sem comprometer a qualidade da avaliação diagnóstica. O preparo intestinal foi eficiente na maioria dos casos $(88 \%)$, com raros efeitos colaterais, tendo sido bem tolerado pelos pacientes.

\section{CONCLUSÃO}

Os resultados obtidos no nosso estudo permitem concluir que os pacientes preferem a CTC à colonoscopia e que não há relação estatística entre a distensão colônica, a qualidade do preparo intestinal (fluido residual) e a preferência do paciente ou seu grau de desconforto. A CTC pode ser considerada uma alternativa à colonoscopia diagnóstica, com vantagens relacionadas à sua comodidade e aceitação dos pacientes.

\section{REFERÊNCIAS}

1. Brasil. Ministério da Saúde. Instituto Nacional de Câncer. Estimativa 2010: incidência de câncer no Brasil. Rio de Janeiro, RJ: INCA; 2009.
2. Levin B, Lieberman DA, McFarland B, et al. Screening and surveillance for the early detection of colorectal cancer and adenomatous polyps, 2008: a joint guideline from the American Cancer Society, the US Multi-Society Task Force on Colorectal Cancer, and the American College of Radiology. CA Cancer J Clin. 2008;58:130-60.

3. Weitzman ER, Zapka J, Estabrook B, et al. Risk and reluctance: understanding impediments to colorectal cancer screening. Prev Med. 2001;32: 502-13.

4. Winawer SJ, Stewart ET, Zauber AG, et al. A comparison of colonoscopy and double-contrast barium enema for surveillance after polypectomy. National Polyp Study Work Group. N Engl J Med. 2000;342:1766-72.

5. Lieberman DA. Cost-effectiveness model for colon cancer screening. Gastroenterology. 1995; 109:1781-90

6. Vernon SW. Participation in colorectal cancer screening: a review. J Natl Cancer Inst. 1997; 89:1406-22.

7. Pickhardt PJ, Choi JR, Hwang I, et al. Computed tomographic virtual colonoscopy to screen for colorectal neoplasia in asymptomatic adults. $\mathrm{N}$ Engl J Med. 2003;349:2191-200.

8. Cummings LC, Cooper GS. Colorectal cancer screening: update for 2011. Semin Oncol. 2011; 38:483-9.

9. von Wagner C, Smith S, Halligan S, et al. Patient acceptability of CT colonography compared with double contrast barium enema: results from a multicentre randomised controlled trial of symptomatic patients. Eur Radiol. 2011;21:2046-55.

10. Graser A, Stieber P, Nagel D, et al. Comparison of CT colonography, colonoscopy, sigmoidoscopy and faecal occult blood tests for the detection of advanced adenoma in an average risk population. Gut. 2009;58:241-8.

11. Svensson MH, Svensson E, Lasson A, et al. Patient acceptance of CT colonography and conventional colonoscopy: prospective comparative study in patients with or suspected of having colorectal disease. Radiology. 2002;222:337-45.

12. Leard LE, Savides TJ, Ganiats TG. Patient preferences for colorectal cancer screening. J Fam Pract. 1997;45:211-8.

13. Winawer SJ, Fletcher RH, Miller L, et al. Colorectal cancer screening: clinical guidelines and rationale. Gastroenterology. 1997;112:594-642.

14. Heresbach D, Barrioz T, Lapalus MG, et al. Miss rate for colorectal neoplastic polyps: a prospective multicenter study of back-to-back video colonoscopies. Endoscopy. 2008;40:284-90.

15. Pickhardt PJ, Kim DH. Colorectal cancer screening with CT colonography: key concepts regarding polyp prevalence, size, histology, morphology, and natural history. AJR Am J Roentgenol. 2009;193:40-6.

16. von Wagner C, Knight K, Halligan S, et al. Patient experiences of colonoscopy, barium enema and CT colonography: a qualitative study. $\mathrm{Br} \mathrm{J}$ Radiol. 2009;82:13-9.

17. Ristvedt SL, McFarland EG, Weinstock LB, et al Patient preferences for CT colonography, conventional colonoscopy, and bowel preparation. Am J Gastroenterol. 2003;98:578-85.

18. Akerkar GA, Yee J, Hung R, et al. Patient experience and preferences toward colon cancer screening: a comparison of virtual colonoscopy and conventional colonoscopy. Gastrointest Endosc. 2001;54:310-5.

19. Zalis ME, Barish MA, Choi JR, et al. CT colono graphy reporting and data system: a consensus proposal. Radiology. 2005;236:3-9. 